\title{
Escenarios innovadores en Educación Física: El trabajo de la expresión corporal y musical mediado por la robótica \\ Innovative scenarios in Physical Education: body and musical expression work mediated by robotics

\author{
Jesús López Belmonte, Santiago Pozo Sánchez, Arturo Fuentes Cabrera, Manuel Ricardo Vicente Bújez \\ Universidad de Granada (Granada)
}

\begin{abstract}
Resumen: La incipiente revolución tecnológica, instaurada en nuestro actual sistema educativo, está originando una optimización en el proceso de enseñanza-aprendizaje por parte de los discentes, siendo la robótica una de las áreas innovadoras que se han incorporado a la docencia. Este estudio pretende conocer la influencia de la robótica sobre diversos indicadores académicos en contenidos relacionados con la expresión corporal y musical en la etapa de Educación Secundaria Obligatoria. Para ello, se ha seguido un diseño descriptivo y correlacional a través de un método cuantitativo, en una muestra de 210 alumnos, correspondiente a los distintos cursos que engloba esta etapa educativa, a los que se le aplicó un cuestionario ad hoc tras la realización de una experiencia innovadora con coreografías musicales robotizadas. Los resultados revelan que la utilización de recursos robóticos ha originado altos índices en cuanto a motivación, atención, participación, colaboración, clima de aula y competencia digital, encontrando diferencias estadísticamente significativas en función del sexo y curso del alumnado.
\end{abstract}

Palabras clave: TIC, innovación educativa, robótica, expresión corporal, educación física.

Abstract: The incipient technological revolution, established in our current educational system, is triggering an optimization in the teaching-learning process by the students, robotics being one of the innovative areas having been incorporated into teaching. This study aims to know the influence of robotics in the learning of corporal and musical expression in the stage of Compulsory Secondary Education. To do that, a descriptive and correlational design was implemented on the basis of the quantitative methodology. The sample consisted of 210 students from different courses included at this educational stage, who filled in an ad-hoc questionnaire after the completion of an innovative experience with robotic musical choreography. The results reveal that the use of robotic resources originated high rates in terms of motivation, attention, participation, collaboration, classroom climate, and digital competence, with statistically significant differences by students' gender and course.

Keywords: ICT, educational innovation, robotics, body expression, physical education.

\section{Introducción}

Las tecnologías de la información y la comunicación (TIC) ocupan un lugar de enorme importancia en la cotidianeidad de la sociedad actual. Apenas sin percatarnos de ello, cada día utilizamos una ingente cantidad de dispositivos tecnológicos que facilitan nuestras acciones diarias (Maldonado, García \& Sampedro-Requena, 2019).

La incorporación de las TIC en nuestras vidas se ha producido en base a un vertiginoso proceso de adaptación favorecido por los enormes avances obtenidos en el campo de la tecnología (Rodríguez, Cáceres \& Alonso, 2018). De esta forma, se ha producido un paso de la era analógica a la digital, una era en la que casi la totalidad de los individuos que componen la sociedad utiliza herramientas tecnológicas para cubrir sus necesidades básicas (Jódar, 2010; Viñals \& Cuenca, 2016).

En consecuencia, cada individuo ha sido sometido de forma forzosa a un proceso de alfabetización digital, siendo los más jóvenes los que han llevado a cabo dicho proceso con mayor rapidez, ya que - para ellos- la tecnología resulta altamente atractiva y novedosa (Area, 2015; Castellanos, Sánchez \& Calderero, 2017). Estos jóvenes utilizan herramientas tecnológicas de manera habitual, al haber nacido en el seno de una sociedad invadida por las TIC y al haber aprendido a utilizarlas de forma prácticamente instintiva (Rodríguez, Romero \& Campos, 2018). Todo lo contrario su-

Fecha recepción: 11-01-20. Fecha de aceptación: 24-03-20

Jesús López Belmonte

jesuslopez@ugr.es cede con los inmigrantes digitales, entendida como una población de edad más avanzada que necesita un esfuerzo importante para incluir en su vida cotidiana las herramientas que los nativos digitales emplean con soltura y destreza (Román, Almansa \& Cruz, 2016; Sánchez \& Castro, 2013).

En el ámbito concreto de la educación se ha experimentado una gran revolución tecnológica debido a los constantes avances producidos en materia educativa como consecuencia del continuo progreso de una sociedad cada vez más tecnificada (Colina \& Túa, 2018). En este sentido, no debemos obviar la importancia del enfoque pedagógico/ metodológico con el que se apliquen las TIC en el seno del proceso de enseñanza-aprendizaje, ya que existen evidencias (Sáez-López \& Sevillano-García, 2017) de que la tecnología per sé no produce mejoras educativas, sino que depende del cómo se apliquen estas y de su capacidad para poder alcanzar aprendizajes significativos de una forma natural y dinámica. Desde este paradigma innovador, una opción puede ser enseñar contenidos tradicionales mediante un enfoque metodológico o modelo pedagógico novedoso centrándose en la búsqueda de nuevos contenidos a impartir incorporándose actividades innovadoras que favorezcan y acentúen un nuevo papel del alumnado mucho más colaborativo, activo y creativo (Carriedo, Méndez, Fernández \& Ceccini, 2020). En este sentido, las tecnologías de la información y de la comunicación (TIC) pueden ofrecernos multitud de recursos relacionados con la expresión corporal y musical, que atraen la atención de niños y adolescentes y conectan con sus intereses y motivaciones.

Por ello, resulta fundamental que las instituciones educativas potencien políticas para incluir las TIC en los espa- 
cios formativos (Ortega, 2009; Quezada, Teijeiro, Riofrío \& Brito, 2018), acción que requiere que los centros estén correctamente equipados para poder llevar a cabo una óptima inclusión de equipamiento tecnológico y dispositivos digitales en sus aulas (Méndez \& Delgado, 2016).

A nivel normativo, el corpus legislativo vigente también hace alusión directa a la importancia de las TIC dentro del proceso de enseñanza aprendizaje. El Real Decreto 1105/2014, de 26 de diciembre, por el que se establece el currículo básico de la Educación Secundaria Obligatoria y del Bachillerato, establece -en su capítulo II- que los discentes de Educación Secundaria Obligatoria tendrán como objetivo «adquirir una preparación básica en el campo de las tecnologías, especialmente las de la información y la comunicación» (Real Decreto 1105/2014,p.177).

La citada norma establece-además- que el correcto desarrollo de la competencia digital en los discentes requiere de una óptima incorporación de las TIC a las aulas, en consonancia con la cultura digital imperante, para que el alumnado pueda integrar y correlacionar sus aprendizajes con el mundo digital actual y con nuevo mundo interconectado.

Siguiendo con el análisis de la legislación vigente en materia educativa, la Ley Orgánica 8/2013, de 9 de diciembre, para la mejora de la calidad educativa (LOMCE), establece en su Preámbulo que las TIC conformarán una pieza clave para llevar a cabo las modificaciones necesarias en el paradigma de las metodologías de aprendizaje, partiendo del hecho de que «el uso responsable y ordenado de estas nuevas tecnologías por parte de los alumnos y alumnas debe estar presente en todo el sistema educativo» (Ley Orgánica 8/2013, p.9). Además, cabe destacar que las TIC se trabajarán de forma interdisciplinar y transversal fomentando su utilización como un recurso pedagógico y metodológico eficiente constituyéndose, así, en un desafío para el sistema educativo al comprometer al alumnado y al profesorado a interactuar con el entorno social y cultural en el que desarrolla su actividad asumiendo un nuevo enfoque educativo (Schleicher, 2015) que permita aprender a aprender, crear pensadores, resolver problemas, trabajar de forma cooperativa y colaborativa desarrollando la capacidad de expresión y comunicación (Vicente \& Díaz, 2012). En definitiva, implica la ruptura entre el aprendizaje formal e informal, permitiendo un modo más social de aprender, donde supone el paso del aprendizaje basado en el currículum al aprendizaje basado en problemas (SáezLópez, Sevillano-García \& Pascual-Sevillano, 2019).

\section{Consideraciones sobre la robótica educativa y el Makey Makey}

En particular, una de las innovaciones emergentes que en el campo de la educación ha asumido un enorme impulso -propiciado por los enormes avances experimentados en el campo de la tecnología- es la robótica (Camilleri, 2017; López, Pozo, Fuentes \& Romero, 2019a; Yu-mei, Qing, Jian-tong, Huan \& Jia, 2017).

A nivel educativo, la robótica va cobrando cada vez más importancia dentro de los espacios de aprendizaje, especialmente en la etapa de educación secundaria (Alimisis, 2014; Benitti, 2012; Mouhaffel, 2018; Vega, Cufié, Rueda \& Llinás, 2016). Su utilización como recurso pedagógico permite fo- mentar la mejora de determinados indicadores educativos como las relaciones sociales de los alumnos, la autonomía, la creatividad, la atención y generando contextos de aprendizaje motivadores para que los discentes busquen de forma autorregulada soluciones y alternativas a las distintas cuestiones que se plantean en el aula (Bravo \& Guzmán, 2012; García, 2015; Vivas \& Sáez, 2019).

Asimismo, se ha constatado que el empleo de la robótica a nivel didáctico permite desarrollar especialmente la competencia digital, pero también contribuye al trabajo de otros aspectos como la motivación, el interés, la participación, el trabajo cooperativo, el autoaprendizaje, la actitud crítica, la elaboración colaborativa del conocimiento, el acceso a distintas vías de información, la aplicación práctica del conocimiento, la creatividad (Román, Hervás \& Guisado, 2017) e incluso la expresión de sentimientos y desarrollo de las emociones de los discentes (López, Pozo, Vicente \& Díaz, 2019).

Pedagógicamente, la robótica desarrolla en el estudiantado un pensamiento computacional, entendido como una de las destrezas más importantes de la educación de la sociedad de la información y el conocimiento, con el que se consigue un procesamiento cognitivo de orden superior a través de la abstracción, el empleo de procesos lógicos y la aplicación de algoritmos (González, Estebanell \& Peracaula, 2018). En esta línea, el uso de la robótica supone incentivar en el alumnado el poder de la imaginación al tener que indagar e idear una solución ante un problema que se le plantea y con los recursos disponibles con los que cuenta (GarcíaVarcárcel \& Caballero, 2019), sus capacidades artísticas plasmadas en el diseño robótico, sus habilidades manipulativas con la construcción del propio robot y trabajo cognitivo mediante la realización de la programación para que todo el ensamblaje efectuado tenga sentido y cumpla con su finalidad(García, 2015).

Uno de los dispositivos robóticos que se pueden emplear en el terreno educativo es el Makey Makey (en adelante, MM). Enriquez (2018) lo define como una plataforma multimedia que permite controlar cualquier dispositivo informático sin necesidad de tener conocimientos electrónicos ni de programación. El dispositivo fue creado por Jay Silver y Eric Rosenbaum, dos estudiantes estadounidenses del laboratorio tecnológico de Massachusetts, en EE.UU (Román, et al., 2017).

La tecnología del dispositivo MM está formada por varios componentes (Chien \& Yu, 2014; Hagerman, 2017; Lee, Kafai, Vasudevan \& Davis, 2014). En la parte superior se localiza la entrada a puerto USB para conectar el dispositivo a un ordenador. La parte frontal (figura 1) contiene las funciones necesarias para controlar el objeto con el que se pretende interactuar. En el reverso (figura 2) se encuentran integrados el procesador y los pines de la placa controladora programada en lenguaje Arduino, a través del estándar $\mathrm{C}++$, permitiendo realizar cambios en la programación del disposi-

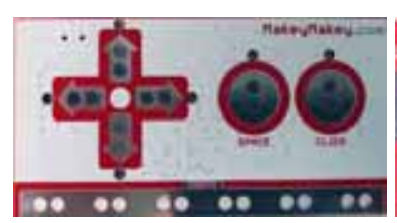
Fuente: Elaboración propia
Figura 1. Placa controladora (frontal)

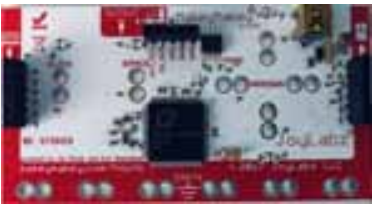

Figura 2. Placa controladora (reverso) Fuente: Elaboración propia 
tivo. Por último, el cableado y las pinzas de control se conectan en las distintas hendiduras destinadas para tal fin tanto en la parte frontal como en la trasera.

Con respecto a su aplicación práctica, Dougherty (2012) afirma que el dispositivo MM presenta enormes potencialidades para ser utilizado en los centros educativos. Su puesta en escena depende en gran medida de la actitud del profesorado y de sus propuestas y proyectos para emprender una acción formativa de carácter innovador de la mano de la robótica. Todo ello con el propósito de alcanzar únicas y fascinantes experiencias de aprendizaje que lleven al colectivo discente a tomar las riendas de la acción instructiva y genere por sí mismo el conocimiento, compartido por el resto de estudiantes y guiado por el docente (Jason \& Jammie, 2019; Mäkelä \& Vellonen, 2018).

Varios estudios basados en experiencias innovadoras con dicho recurso robótico han constatado que contribuye a la concentración y a la motivación, además de potenciar el aprendizaje cooperativo, lo que propicia que mejoren las relaciones dentro del grupo de iguales y se contribuya a la consecución de aprendizajes significativos (Lozano, Guerrero \& Gordillo, 2016) además de mejorar los resultados de las pruebas de evaluación, ya que los alumnos salen del aprendizaje convencional, se encuentran más motivados y trabajan con mayor nivel de autonomía (Chaves, Esquivel, Jiménez \& Sánchez, 2018).

Una experiencia robótica para el fomento de la expresión musical y corporal

En el ámbito de la educación musical y de la expresión corporal, debemos destacar el papel de la música al constituirse como un elemento clave para la expresividad, la transmisión de emociones y por encontrarse indisolublemente unida al movimiento (Folch, Córdoba, \& Ribalta, 2020). Así, por ejemplo, al escuchar una melodía muy rítmica, de forma natural marcamos el ritmo con los pies, con las manos o la cabeza. El cuerpo se mueve de forma instintiva, primitiva, donde la unión entre música y movimiento suponen un medio de expresión idóneo en el que, además, se desarrollan esquemas propios de relación espacial y temporal (Bautista, Tan, Ponnusamy \& Yau, 2015). Diversos autores han defendido que las TIC pueden servir de suplemento y aliciente para optimizar la enseñanza de dichas materias (Eyles, 2018; Hernández, Cardona \& Hernández, 2016; Giráldez, 2010; Ruismäki \& Juvonen, 2009). Existen contenidos concretos del ámbito musical que pueden verse altamente beneficiados por la implementación de las TIC, siendo especialmente propicios para tal fin los contenidos relacionados con el movimiento y la expresión corporal (De Castro, 2015) así como la creación e improvisación musical (Serrano, 2017). Por su parte, Thaer (2016) considera que la mayor parte de las TIC son válidas para potenciar el aprendizaje de los elementos teóricos de la música y para propiciar espacios innovadores que faciliten la creatividad del discente.

Por otro lado, diversos autores han constatado que la realización de actividades dentro del ámbito de la educación física utilizando las TIC como elemento complementario supone un avance crucial para la consolidación de un proceso de enseñanza y aprendizaje que favorezca la puesta en marcha del trabajo activo, participativo, motivador y autónomo
(Trujillo \& Pozo, 2020; Weng, Rathinasabapathi, Weng \& Zagita, 2019; Vicente, Díaz, López \& Pozo, 2019; Hinojo, Mingorance, Trujillo, Aznar \& Cáceres, 2018; Hinojo, López, Fuentes, Trujillo \& Pozo, 2020; Lleixà, 2017; Chacón, Castro, Zurita, Espejo \& Martínez, 2016; Fernández, Herrera \& Navarro, 2015; Monguillot, González \& Guitert, 2015).

En este estudio se ha implementado una unidad didáctica innovadora para trabajar contenidos propios de la expresión corporal y musical como el ritmo, la percepción espaciotemporal, el movimiento y lenguaje corporal, pequeñas composiciones y coreografías a través de TIC en las sesiones de Educación Física de los distintos cursos que abarca la etapa de Educación Secundaria. Dicha unidad didáctica se compuso de ocho sesiones en las que los alumnos tuvieron que realizar coreografias y creaciones musicales a través de la interacción con elementos cotidianos, dispositivos digitales y robóticos que desembocaran en una actividad física cooperativa. Todos los contenidos fueron adaptados según los objetivos didácticos de área de cada curso.

Entre las actividades realizadas en la unidad didáctica se encuentran:

-Jugar a juegos sencillos digitales para la familiarización con el material robótico, cuyo movimiento del avatar es originado por pulsadores de plastilina, conectados a los elementos del MM, separados a una distancia considerable para que se produzca un desplazamiento del participante.

-Experimentar los sonidos de la escala natural y cromática tocando un piano digital subiendo y bajando escaleras. El sonido es producido al pisar el escalón que contiene papel con grafito conectado a los elementos del MM que permiten conducir la respuesta producida con la pisada del estudiante a una aplicación digital.

-Interpretar obstinatos melódicos y rítmicos a través de piezas de fruta conectadas al kit MM, originando cada fruta un tono distinto.

-Efectuar coreografías e improvisar patrones melódicos a través del movimiento y posterior contacto entre los estudiantes que contenían en sus manos las pinzas que transmiten la señal conductora.

Para la realización de las actividades propuestas se proporcionaron diferentes indicaciones y materiales para ayudar a los estudiantes a estructurar su tarea. Asimismo, fue necesario contar con elementos de conducción eléctrica tales como un vaso de agua, papel de aluminio, pieza de fruta, plastilina, dibujo realizado con el grafito del lápiz e incluso el propio cuerpo humano (como principal elemento de expre-

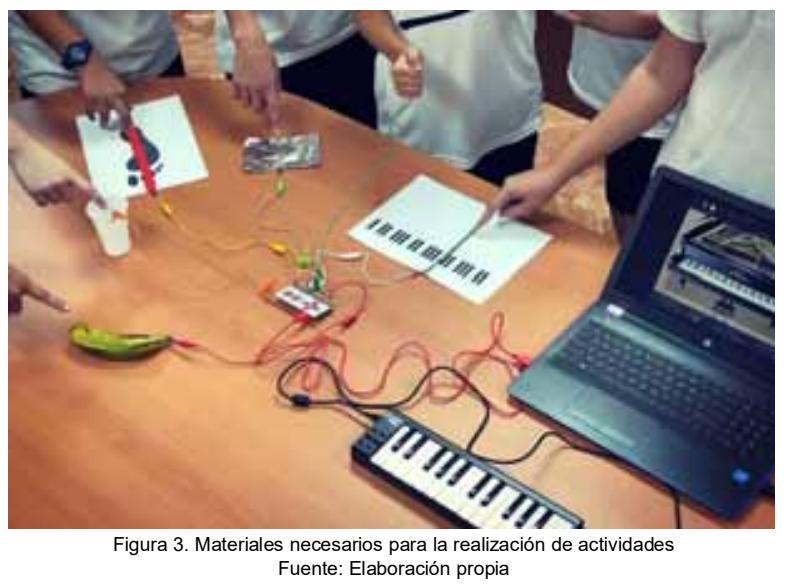


sión), además de un kit de robótica MM, un ordenador portátil, un bafle, un sintetizador y una app musical (Pianissimo), entre los materiales más destacados como se visualiza en la figura 3 .

\section{Justificación y objetivos de la investigación}

La utilización de la tecnología educativa -en general-y de la robótica - en particular- es el foco de interés que presenta este estudio encuadrado dentro del marco innovador que persigue la legislación actual, con la finalidad de fomentar la inclusión de las TIC en los espacios de aprendizaje. Un estudio bibliométrico reciente realizado por Cabrera (2020) refleja el estado de la cuestión de un corpus literario que demanda la acogida de experiencias e investigaciones acerca de la integración de las TIC en Educación Física. Todo ello para seguir la senda establecida anteriormente donde se demuestra la motivación alcanzada en los participantes como consecuencia de la aplicación de recursos tecnológicos (Rivilla, Sillero, Grande, Sampedro \& Gómez, 2014) y la gran fuente de información que proporcionan los medios electrónicos sobre el entorno en el que se utiliza (Reche, Cardona, Díaz, Gómez \& Pino, 2018).

Asimismo, este trabajo pretende fomentar y exportar, como asentaron Ladrón, Cabero \& Almagro (2019), el conocimiento y la praxis del profesorado desde un enfoque tecnológico, pedagógico y disciplinar (TPACK), acorde a los nuevos tiempos y corrientes por las que circulan los procesos de enseñanza y aprendizaje en una sociedad digital.

Estas premisas deben tenerse en cuenta para que los docentes logren despertar el interés y captar la atención de un colectivo estudiantil cada vez más sedentario a causa de la incidencia de la tecnología que ha ocasionado nuevas formas de relación y ocio desde una perspectiva con menor carga física, lo que deriva en un descenso de la actividad y, por consiguiente, un deterioro de la salud de las personas (Santos, et al., 2018). En base a ello, tomando las consideraciones de Chaddha, Jackson, Richardson \& Franklin (2017) y de Suárez et al. (2018) se emplea la tecnología para dinamizar al estudiantado hacia la realización de actividad física centrada en la expresión corporal y musical mediada por recursos robóticos.

El objetivo general que persigue este estudio es conocer la influencia de la robótica sobre diversos indicadores académicos en contenidos relacionados con la expresión corporal y musical en la educación secundaria.

Para determinar el alcance de este objetivo se han formulado los siguientes lineamientos con mayor grado de concreción:

-Averiguar la motivación alcanzada por los estudiantes.

-Conocer el grado de atención de los discentes.

-Conocer la participación del alumnado en las actividades robóticas propuestas.

-Determinar la incidencia de la robótica en el aprendizaje cooperativo.

-Descubrir la influencia de la robótica en el clima escolar de trabajo.

-Averiguar el nivel de competencia digital de los discentes con elementos robóticos.

-Determinar la influencia del sexo de los discentes con respecto a la motivación, atención, participación, aprendiza- je cooperativo, clima escolar y competencia digital.

-Averiguar la asociación del curso al que pertenecen los estudiantes en cuanto a la motivación, atención, participación, aprendizaje cooperativo, clima escolar y competencia digital.

\section{Método}

\section{Diseño de investigación y análisis de datos}

El estudio se ha efectuado por medio de un diseño no experimental de tipo descriptivo y correlacional siguiendo una metodología cuantitativa, bajo las orientaciones de McMillan \& Schumacher (2005).

El análisis de los datos se ha realizado a través del programa Statistical Package for the Social Sciences (SPSS) v.25, tomando un $p<.05$ como diferencia estadísticamente significativa.

En el análisis descriptivo se ha determinado el número de casos obtenidos en cada categoría y el porcentaje correspondiente. Se han utilizado estadísticos como la media (M), la desviación típica(DT), el coeficiente de asimetría de Pearson $\left(\mathrm{CA}_{\mathrm{P}}\right)$ y el coeficiente de apuntamiento de Fisher $\left(\mathrm{CA}_{\mathrm{F}}\right)$. Y para la comparación de variables se ha llevado a cabo la prueba Chi-cuadrado $\left(\div^{\circ}\right)$ y V de Cramer $\left(\mathrm{V}_{\text {Cramer }}\right)$, en base a las consideraciones de Landero \& González (2006).

\section{Participantes}

Este estudio se ha realizado en la etapa de educación secundaria de una cooperativa de enseñanza del sur de España. La elección de esta tipología de centro educativo queda justificada por los estudios realizados por López \& Fuentes (2018) y López, Pozo, Fuentes \& Romero (2019b) en los que se constata la búsqueda de la innovación, eficacia y excelencia educativa que persiguen las cooperativas de enseñanza.

La muestra seleccionada se compone de los discentes matriculados en los cursos $1^{\circ}, 2^{\circ}, 3^{\circ}$ y $4^{\circ}$ de educación secundaria $(n=210)$. En la tabla 1 se especifican las peculiaridades de los estudiantes, relacionadas con el sexo, edad, religión y nacionalidad. Estos sujetos han sido escogidos a través de un muestreo dirigido o por conveniencia, cuya razón se focaliza en la facilidad para acceder a la muestra de alumnos (Hernández, Fernández \& Baptista, 2014).

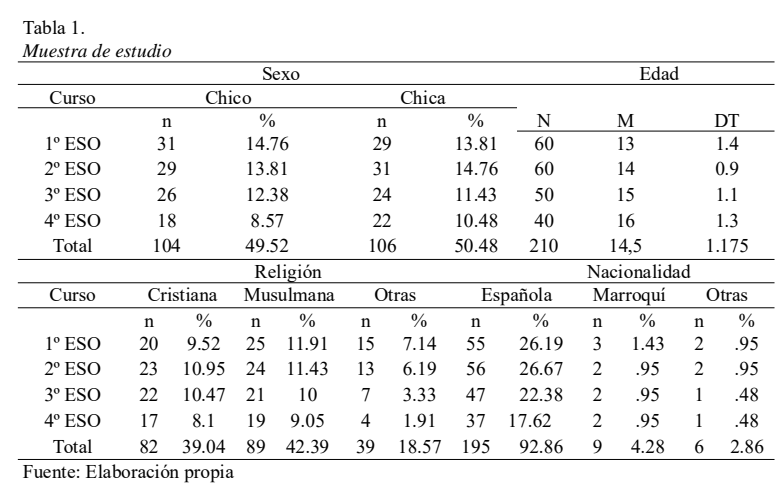

\section{Instrumento}

El proceso de recogida de datos se ha realizado a través de un cuestionario ad hoc que recoge la percepción de los estudiantes sobre las distintas dimensiones que articula. Asimismo, el cuestionario presenta un diseño que permite 
dar respuesta a las distintas necesidades de la investigación. La herramienta se compone de 33 ítems en total, organizados en tres dimensiones: a) Socio-demográfica (6 ítems); b) Experiencia robótica (12 ítems); c) Competencia digital discente (15 ítems). El instrumento sigue un formato de respuesta variado, combinando cuestiones con una valoración en escala tipo Likert con un rango de cuatro posibilidades (1Nada, 2-Poco, 3-Bastante y 4-Totalmente) y otras de respuestas cerradas.

El instrumento se ha validado primeramente de forma cualitativa por método Delphi, conformado por un grupo de 8 expertos en tecnología educativa de diferentes universidades españolas, quienes ofrecieron una opinión favorable del cuestionario y lo retroalimentaron con propuestas de mejora con el propósito de optimizar la herramienta. Los juicios de los especialistas fue analizado mediante las pruebas Kappa de Fleiss y W de Kendall, resultando ser concordantes entre ellos, a la vez que pertinentes $(\mathrm{K}=0.84 ; \mathrm{W}=0.87)$.

A continuación, se efectuó una validación cuantitativa por medio de un análisis factorial exploratorio con el método de componentes principales y rotación varimax. Se determinó dependencia entre las variables definidas con la prueba de esfericidad de Bartlett (2451.39; $p<0.001)$ y una adecuada adecuación muestral con el test de Kaiser-Meyer-Olkin $(\mathrm{KMO}=0.88)$.

Finalmente, se decretó la fiabilidad del cuestionario a través de diferentes estadísticos como el alfa $(\alpha)$ de Cronbach (0.86), la fiabilidad compuesta (0.87) y la varianza media extractada (0.84).

\section{Dimensiones y variables de estudio}

En este apartado se concretan las dimensiones utilizadas en la investigación, así como las variables que articulan junto con su abreviatura, con el propósito de amenizar la lectura y facilitar la comprensión e interpretación de los resultados.

a) Socio-demográfica: acoge a los ítems relacionados con el sexo, edad, curso, nacionalidad, ciudad y religión.

b) Experiencia robótica: engloba a los ítems concernientes a la motivación (MOTIV), atención (ATEN), participación (PARTI), aprendizaje cooperativo de los estudiantes (ACOOP) y clima originado en los espacios de aprendizaje (CLIM), como consecuencia de la aplicación de la experiencia didáctica basada en la robótica.

c) Competencia digital discente: abarca a los ítems alusivos a la valoración de la competencia digital (CD) en sus distintas áreas.

\section{Procedimiento}

El estudio tuvo su origen en el tercer trimestre del curso académico 2018-2019 con la aceptación por parte del equipo directivo del proyecto presentado por los investigadores en la cooperativa de enseñanza en cuestión. Una vez obtenida la autorización por parte de este ente organizacional, se procedió a iniciar todo el protocolo de investigación que consistió en el desarrollo de una unidad didáctica compuesta por ocho sesiones en la asignatura de Educación Física donde se impartieron los contenidos mediados por la robótica. Una vez concluida tal experiencia innovadora se inició el proceso de recogida de datos a través de un cuestionario previamente validado. Tanto en el proceso de recogida de datos, como en el posterior análisis de los mismos, se respetaron el anonimato de los discentes y se siguieron las pautas éticas para el tratamiento de la información (García, Alfaro, Hernández y Molina, 2006). Por último, se importaron todos los datos en el programa estadístico para obtener información que permitiera dar respuesta a los objetivos estipulados en el estudio y dar origen a nuevas conclusiones sobre el estado de la cuestión analizada.

\section{Resultados}

Abordando los resultados obtenidos en el proceso de investigación, la Tabla 2 muestra los valores obtenidos en las diferentes variables de estudio en base a una escala Likert de 4 puntuaciones y estadísticos específicos. Estos datos revelan que los participantes han alcanzado índices de respuestas positivos en la totalidad de las variables analizadas. Las referentes a la motivación alcanzada por el alumnado y al nivel de competencia digital demostrado en el proceso de enseñanza y aprendizaje mediado por la robótica han sido las dos variables con una puntuación más positiva en comparación con el resto. Por el contrario, se ha podido constatar que la aplicación de la robótica para el tratamiento de los contenidos didácticos no ha generado contextos de trabajo que propicien significativamente el aprendizaje cooperativo, siendo esta variable la que ha alcanzado la puntuación más baja de entre el grupo de variables estudiado.

Tabla 2 .

\begin{tabular}{|c|c|c|c|c|c|c|c|c|}
\hline & \multicolumn{4}{|c|}{ Escala Likert $n(\%)$} & \multicolumn{4}{|c|}{ Parámetros } \\
\hline & Nada & Poco & Bastante & Totalmente & $\mathrm{M}$ & DT & $\mathrm{CA}_{\mathrm{P}}$ & $\mathrm{CA}_{\mathrm{F}}$ \\
\hline MOTIV & $12(5.71)$ & $21(10)$ & $60(28.57)$ & 117 (55.72) & 3.342 & .876 & 2.673 & .651 \\
\hline ATEN & $15(7.14)$ & $85(40.48)$ & $92(43.81)$ & $18(8.57)$ & 2.538 & .751 & 2.051 & -.332 \\
\hline PARTI & $3(1.43)$ & $15(7.14)$ & $93(44.28)$ & $99(47.15)$ & 2.976 & .591 & 3.349 & 2.061 \\
\hline ACOOP & $47(22.38)$ & $63(30)$ & $68(32.38)$ & $32(15.25)$ & 2.405 & .996 & 1.409 & -1.067 \\
\hline CLIM & $12(5.71)$ & $56(26.7)$ & $81(38.57)$ & $61(29.02)$ & 2.909 & .881 & 2.165 & -.762 \\
\hline $\mathrm{CD}$ & $5(2.38)$ & $18(8.57)$ & $92(43.81)$ & $95(45.24)$ & 3.319 & .729 & 3.181 & .716 \\
\hline Total & $94(7.5)$ & $258(20.4)$ & $486(38.6)$ & $422(33.5)$ & 2.981 & .915 & 2.164 & -.578 \\
\hline
\end{tabular}

A partir de los resultados obtenidos en la tabla anterior se ha llevado a cabo una comparativa gráfica que aglutina los valores promedio de las distintas variables de estudio, su valor totalizado y el valor central de la escala Likert (figura 4), para poder tener una perspectiva global de los valores obtenidos. De esta forma, se ha constatado que todas las variables de estudio superan el valor central de la escala $\left(\mathrm{V}_{\text {CENTRAL }}=2.5\right)$, a excepción del mencionado fomento del aprendizaje cooperativo mediante el uso de la robótica. Por otro lado, destacan especialmente las puntuaciones obtenidas en las variables relacionadas con la motivación generada en las tareas propuestas, el grado de atención del alumnado y el nivel de competencia digital discente demos-

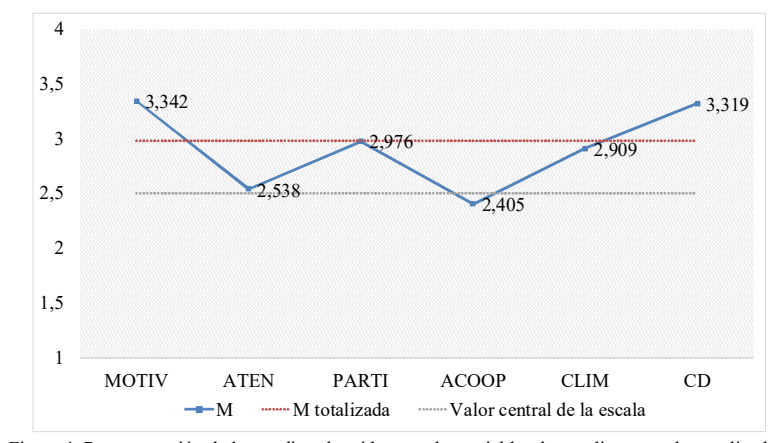

Figura 4. Representación de las medias obtenidas para las variables de estudio y su valor totalizado Fuente: Elaboración propia 
trada en las tareas, situándose en todos los casos por encima del valor de la media totalizada $\left(\mathrm{M}_{\text {TOTALIZADA }}=2.98\right)$.

Con respecto a la influencia del sexo en las distintas variables analizadas (Tabla 3 ), se determina -en base a los resultados alcanzados- que tanto en la atención del alumnado durante el proceso de enseñanza y aprendizaje como en la puesta en práctica del aprendizaje cooperativo se producen diferencias estadísticamente significativas, dado su $p$-valor y el grado de dependencia obtenido en las pruebas realizadas en un nivel de confianza $(Z)$ del $95 \%$ y un margen de error (e) del 5\%. En la variable relacionada con la atención de los discentes ( $p$-valor $=.007 ; \mathrm{V}_{\text {CRAMER }}=.241$ ) es el sexo femenino quien presenta resultados más positivos, mientras que en la variable relacionada con el aprendizaje cooperativo durante el desarrollo de actividades de expresión corporal y musical mediadas por robótica son los chicos quienes obtienen una mayor valoración $\left(p\right.$-valor $\left.=.028 ; \mathrm{V}_{\mathrm{CRAMER}}=.208\right)$. A pesar de los valores obtenidos de significancia estas dos variables, la fuerza de asociación constatada en ambas resulta escasa.

\begin{tabular}{|c|c|c|c|c|c|}
\hline \multirow[b]{2}{*}{ Likert } & \multicolumn{2}{|c|}{ Sexo $n(\%)$} & \multicolumn{3}{|c|}{ Parámetros } \\
\hline & Chico & Chica & $x 2(g l)$ & $p$-valor & $\mathrm{V}_{\text {Crame }}$ \\
\hline MOTIV & & & $.51(3)$ & .918 & .049 \\
\hline Nada & $5(2.38)$ & $7(3.33)$ & & & \\
\hline Poco & $11(5.24)$ & $10(4.76)$ & & & \\
\hline Bastante & $31(14.76)$ & $29(13.81)$ & & & \\
\hline Totalmente & $57(27.14)$ & $60(28.57)$ & & & \\
\hline ATEN & & & $12.24(3)$ & .007 & .241 \\
\hline Nada & $10(4.76)$ & $5(2.38)$ & & & \\
\hline Poco & $52(24.76)$ & $33(15.71)$ & & & \\
\hline Bastante & $36(17.14)$ & $56(26.67)$ & & & \\
\hline Totalmente & $6(2.86)$ & $12(5.71)$ & & & \\
\hline PARTI & & & $2.07(3)$ & .558 & .099 \\
\hline Nada & $1(.48)$ & $2(.95)$ & & & \\
\hline Poco & $7(3.33)$ & $8(3.81)$ & & & \\
\hline Bastante & $51(24.28)$ & $42(20)$ & & & \\
\hline Totalmente & 45 (21.43) & $54(25.71)$ & & & \\
\hline ACOOP & & & $9.07(3)$ & .028 & .208 \\
\hline Nada & $18(8.57)$ & $29(13.81)$ & & & \\
\hline Poco & $26(12.38)$ & $37(17.62)$ & & & \\
\hline Bastante & $39(18.57)$ & $29(13.81)$ & & & \\
\hline Totalmente & $21(10)$ & $11(5.24)$ & & & \\
\hline CLIM & & & $4.15(3)$ & .246 & .140 \\
\hline Nada & $9(4.28)$ & $3(1.43)$ & & & \\
\hline Poco & $25(11.91)$ & $31(14.76)$ & & & \\
\hline Bastante & $42(20)$ & $39(18.57)$ & & & \\
\hline Totalmente & $28(13.33)$ & $33(15.71)$ & & & \\
\hline $\mathrm{CD}$ & & & $1.56(3)$ & .669 & .086 \\
\hline Nada & $2(.95)$ & $3(1.43)$ & & & \\
\hline Poco & $7(3.33)$ & $11(5.23)$ & & & \\
\hline Bastante & $49(23.33)$ & $43(20.48)$ & & & \\
\hline Totalmente & $46(21.91)$ & $49(23.33)$ & & & \\
\hline
\end{tabular}

\begin{tabular}{|c|c|c|c|c|c|c|c|c|}
\hline \multirow[b]{2}{*}{ Likert } & \multicolumn{3}{|c|}{ Curso $n(\%)$} & \multicolumn{5}{|c|}{ Parámetros } \\
\hline & $1^{\circ} \mathrm{ESO}$ & $2^{\circ} \mathrm{ESO}$ & $3^{\circ} \mathrm{ESO}$ & $4^{\circ} \mathrm{ESO}$ & $x 2(g l)$ & $p$-valor & Cont. & $\mathrm{V}_{\text {Cramer }}$ \\
\hline MOTIV & & & & & $3.89(9)$ & .919 & .135 & .236 \\
\hline Nada & $4(1.91)$ & $3(1.43)$ & $3(1.43)$ & $2(.95)$ & & & & \\
\hline Poco & $6(2.86)$ & $7(3.33)$ & $5(2.38)$ & $3(1.43)$ & & & & \\
\hline Bastante & $20(9.52)$ & $12(5.71)$ & $15(7.14)$ & $13(6.19)$ & & & & \\
\hline Totalmente & $30(14.28)$ & $38(18.01)$ & $27(12.86)$ & $22(10.48)$ & & & & \\
\hline ATEN & & & & & $11.77(9)$ & .227 & .23 & .41 \\
\hline Nada & $3(1.43)$ & $4(1.91)$ & $5(2.39)$ & $3(1.43)$ & & & & \\
\hline Poco & $20(9.52)$ & $23(10.95)$ & $19(9.05)$ & $23(10.95)$ & & & & \\
\hline Bastante & 34 (16.19) & $26(12.38)$ & $20(9.52)$ & $12(5.71)$ & & & & \\
\hline Totalmente & $3(1.43)$ & $7(3.33)$ & $6(2.86)$ & $2(.95)$ & & & & \\
\hline PARTI & & & & & $1.14(9)$ & .0 .999 & .073 & .128 \\
\hline Nada & $1(.48)$ & $1(.48)$ & $1(.48)$ & $0(.00)$ & & & & \\
\hline Poco & $5(2.39)$ & $4(1.91)$ & $3(1.43)$ & $3(1.43)$ & & & & \\
\hline Bastante & $26(12.38)$ & $27(12.86)$ & $23(10.95)$ & $17(8.09)$ & & & & \\
\hline Totalmente & $28(9.05)$ & $28(9.05)$ & $23(10.95)$ & $20(9.52)$ & & & & \\
\hline ACOOP & & & & & $41.46(9)$ & $<.001$ & .406 & .770 \\
\hline Nada & $5(2.39)$ & $4(1.91)$ & $18(8.57)$ & $20(9.52)$ & & & & \\
\hline Poco & $22(10.48)$ & $19(9.05)$ & $12(5.71)$ & $10(4.76)$ & & & & \\
\hline Bastante & $24(11.43)$ & $26(12.38)$ & $11(5.24)$ & $7(3.33)$ & & & & \\
\hline Totalmente & $9(4.28)$ & $11(5.24)$ & $9(4.28)$ & $3(1.43)$ & & & & \\
\hline CLIM & & & & & $2.396(9)$ & .984 & .106 & .185 \\
\hline Nada & $3(1.43)$ & $2(.95)$ & $4(1.91)$ & $3(1.43)$ & & & & \\
\hline Poco & $15(7.14)$ & $17(8.09)$ & $14(6.67)$ & $10(4.76)$ & & & & \\
\hline Bastante & $23(10.95)$ & $22(10.48)$ & $20(9.52)$ & $16(7.62)$ & & & & \\
\hline Totalmente & $19(9.05)$ & $19(9.05)$ & $12(5.71)$ & $11(5.24)$ & & & & \\
\hline CD & & & & & $25.21(9)$ & . 003 & .327 & .601 \\
\hline Nada & $1(.48)$ & $1(.48)$ & $2(.95)$ & $1(.48)$ & & & & \\
\hline Poco & $2(.95)$ & $6(2.86)$ & $7(3.33)$ & $3(1.43)$ & & & & \\
\hline Bastante & $20(9.52)$ & $19(9.05)$ & $29(13.81)$ & $24(11.43)$ & & & & \\
\hline Totalmente & $37(17.61)$ & $34(16.19)$ & $12(5.71)$ & $12(5.71)$ & & & & \\
\hline
\end{tabular}

Por último, en la Tabla 4 se analiza la influencia entre el curso del discente y las diferentes variables tomadas en este estudio. Se reflejan diferencias estadísticamente significativas en la variable relacionada con el aprendizaje cooperativa $(p$-valor $=<.001)$ y en el nivel de competencia digital de los discentes ( $p$-.003), en un $Z=1.96 ; e=.05$. De esta forma, se ha constatado que el segundo curso de Educación Secundaria Obligatoria presenta un mayor grado de cooperación que el resto $\left(\mathrm{V}_{\text {CRAMER }}=.770\right)$ en la puesta en práctica de actividades relacionadas con la expresión corporal-musical mediadas por robótica. Por otra parte, ha sido el alumnado del primer curso de Educación Secundaria Obligatoria el que ha mostrado niveles más altos de competencia digital durante el desarrollo de las actividades $\left(\mathrm{V}_{\text {CRAMER }}=.601\right)$. La fuerza de asociación constatada para dichas variables es elevada en ambos casos.

\section{Discusión}

En base a los resultados obtenidos en el proceso investigador se demuestra, como estableció Area (2015), que el uso de la tecnología en la educación produce un componente de atracción reflejado en los valores alcanzados en esta investigación. Coincidiendo con Ortega (2009), en una época marcada por la incidencia que la tecnología efectúa sobre las personas en sus quehaceres cotidianos, resulta imprescindible fomentar y desarrollar las TIC dentro del ámbito escolar, sobretodo en alumnos acostumbrados al uso diario de estos dispositivos como se aclara en Maldonado et al. (2019). Además, cabe destacar que los alumnos de este estudio son nativos digitales, que han aprendido de manera instintiva, como se indica en Rodríguez et al. (2018), que manejan estas herramientas con soltura y destreza, coincidiendo con lo indicado por Román et al. (2016). Es por ello por lo que se despliega esta experiencia innovadora con la finalidad de satisfacer las necesidades de un alumnado nativo digital y mejorar su competencia digital, tal y como establecen Sánchez \& Castro (2013), todo ello fruto del continuo progreso y la sociedad tecnificada, como indican Colina \& Tua (2018), y con la intención de fomentar el uso de TIC en el devenir cotidiano docente y el trabajo discente, como ya señalan Quezada et al. (2018). Una de las herramientas propicias para este tipo de trabajos, como indican López et al. (2019b), es la robótica, base que sustenta este trabajo.

El empleo de recursos TIC provoca en los procesos formativos una optimización del aprendizaje como manifestaron Giráldez (2010) y Ruismäki \& Juvonen (2009), alcanzando índices positivos en la motivación, atención, participación, aprendizaje cooperativo, clima escolar y la competencia digital, tal y como se ha obtenido en este estudio. Al igual que los resultados alcanzados por De Castro (2015) y Serrano (2017), el trabajo de contenidos relacionados con la expresión corporal y musical tales como el ritmo, la percepción espacio-temporal, el movimiento, el lenguaje corporal, pequeñas composiciones y coreografías se han visto beneficiados por medio de las TIC, añadiendo un componente dinamizador en los contenidos impartidos.

En cuanto a la participación, como ya determinaron Chacón et al. (2016), Fernández et al. (2015) y Monguillot et al. (2015), se ve incrementada debido a la influencia que oca- 
siona la tecnología sobre los discentes, provocando un aumento del trabajo activo que deriva en un ambiente motivador, como obtuvo Lleixà (2017). La utilización de recursos didácticos basados en la robótica, como constataron Bravo \& Guzmán (2012) y García (2015), ha reflejado valores positivos en la motivación y atención discente, así como en el entorno o clima de trabajo en el que se efectúan los procesos de enseñanza y aprendizaje. El hecho de efectuar una metodología instructiva fundamentada en la robótica supone una mejora paulatina de la competencia digital y del aprendizaje cooperativo de los discentes, como verificaron en estudios recientes Román et al. (2017) y que se produce en esta investigación. Concretamente, el recurso MM utilizado en las diferentes sesiones llevadas a cabo durante la experiencia ha supuesto, en términos generales, una potenciación de la motivación y del aprendizaje cooperativo como ya alcanzaron anteriormente Lozano et al. (2016). Además, se fomenta y se alcanzan altas cotas de participación, ya señalado en otros estudios como Jason \& Jammie (2019) y Mäkela \& Vellonen (2018). Todo ello, como hemos señalado, coincide con lo establecido por Chaves et al. (2018), con un importante incremento de la citada la motivación.

\section{Conclusiones}

La sociedad está fuertemente condicionada por los avances tecnológicos que hoy día están presentes en la vida de las personas. El estudio realizado verifica que la utilización de recursos TIC, específicamente elementos propios de la robótica, alteran distintas variables propias de los procesos de enseñanza que se dan cita en la actualidad. Aspectos como la motivación, atención, participación, colaboración, clima de aula y competencia digital, que han sido analizados en esta investigación, se han visto favorecidos considerablemente gracias al empleo de herramientas innovadoras que los docentes incorporan en su praxis diaria con la finalidad potenciar y optimizar los tiempos de aprendizaje.

Particularmente, la robótica ha permitido un alto impacto en la motivación y en la competencia digital del alumnado derivado de un aprendizaje mediado por la tecnología.

Tomando de referencia el sexo de los participantes, en base a las pruebas estadísticas llevadas a cabo ( $x 2(\mathrm{gl}), \mathrm{p}$ valor y $\left.\mathrm{V}_{\text {Cramer }}\right)$, se han encontrado diferencias estadísticamente significativas en el aprendizaje cooperativo y en la atención de los discentes. La robótica ha originado en los chicos un mayor alcance en la cooperación, reflejada en el aprendizaje que han desarrollado durante las diversas sesiones efectuadas. Sin embargo, en las chicas ha propiciado efectos positivos en la atención manifestada durante el trabajo de los contenidos y en las actividades realizadas.

Aunque a nivel estadístico el sexo de los participantes no ha alcanzado más diferencias significativas, se determina que el resto de variables analizadas en este estudio (motivación, participación, clima de trabajo y competencia digital) se han visto favorecidas por la innovación robótica efectuada en las sesiones de Educación Física.

Con respecto al curso en el que se encuentran los discentes, las diferencias estadísticamente significativas se han vuelto a dar en el aprendizaje cooperativo, además de la competencia digital de los estudiantes. En el curso segundo de educación secundaria, el uso de la robótica ha potenciado un aprendizaje en colaboración de los distintos perfiles que componen el grupo de alumnos de aula. Mientras que en primero de educación secundaria la robótica ha permitido optimizar la competencia digital de los participantes. Al igual que ha sucedido con el sexo de los participantes, en las demás variables no significativas a nivel estadístico la utilización de recursos tecnológicos en los distintos cursos ha propiciado el alcance de valoraciones favorecedoras en los discentes.

Este estudio concluye que la robótica ha ocasionado adecuados valores en los diversos indicadores académicos tenidos en consideración en esta investigación, reforzando así los hallazgos encontrados en estudios previos sobre la incidencia tecnológica en la educación actual.

Como prospectiva de esta investigación se pretende expandir en la comunidad científica los hallazgos y las potencialidades aquí encontradas con el propósito de incentivar la difusión de prácticas innovadoras en una educación física que requiere de la incorporación de nuevas formas y medios para el alcance de los objetivos didácticos, competencias y el trabajo de los diferentes contenidos que establece el currículum oficial. Este estudio sitúa a la robótica como una tecnología emergente adecuada para la acción formativa en educación física, generando nuevos caminos para la construcción compartida del conocimiento y de la mano de recursos electrónicos propios de una sociedad cada vez - a edades tempranas-más familiarizada con la tecnología.

La principal limitación de este estudio se centra en la focalización de la muestra en un solo centro escolar, de una única tipología y etapa educativa. Como futura línea de investigación se pretende replicar esta investigación en distintos tipos de centros educativos con la finalidad de realizar un estudio comparativo entre centros públicos, privados y concertados en relación a la influencia que ocasiona la tecnología educativa en los nativos digitales.

\section{Referencias}

Alimisis, D., Moro, M., Arlegui, J., Pina, A., Frangou, S., \& Papanikolaou, K. (2007). Robostics and constructivism in education: the TERECoP project. European Logo, 40, 1-11.

Area, M. (2015). La alfabetización digital y la formación de la ciudadanía del siglo XXI. Revista Integra Educativa, 7(3), 2133.

Bautista, A., Tan, L., Ponnusamy, L., \& Yau, X. (2015). Curriculum integration in arts education: connecting multiple art forms through the idea of 'space'. Journal of Curriculum Studies, 48(5), 610-629. https://doi.org/10.1080/ 00220272.2015 .1089940

Benitti, F. (2012). Exploring the educational potential of robotics in schools: A systematic review. Computers y Education, 58(3), 978-988.

Bisquerra, R. (2004). Metodología de la investigación educativa. Madrid: La Muralla.

Bravo, F. Á., \& Guzmán, A. (2012). La robótica como un recurso para facilitar el aprendizaje y desarrollo de competencias generales. Teoría de la Educación. Educación y Cultura en la Sociedad de la Información,13(2), 120-136. Recuperado de: http:// campus.usal.es/ revistas_trabajo/index.php/revistatesi/article/ vie w/9002/9247 
Cabrera, J. F. (2020). Producción científica sobre integración de TIC a la Educación Física. Estudio bibliométrico en el periodo 1995-2017. Retos, 37(37), 748-754.

Camilleri, P. (2017). Minding the Gap. Proposing a Teacher Learning-Training Framework for the Integration of Robotics in Primary Schools. Informatics in Education, 16(2), 165-179.

Carriedo, A., Méndez-Giménez,A., Fernández-Río, J., \& Cecchini., J. (2020). Nuevas posibilidades y recursos para la enseñanza de la expresión corporal en educación física: internet y los retos virales. Retos, 37, 722-730.

Castellanos, A., Sánchez, C., \& Calderero, J. F. (2017). Nuevos modelos tecnopedagógicos. Competencia digital de los alumnos universitarios. Revista electrónica de investigación educativa, 19(1), 1-9. http://dx.doi.org/10.24320/ redie.2017.19.1.1148

Chacón, R., Castro, M., Zurita, F., Espejo, T., \& Martínez, A. (2016). Videojuegos activos como recurso TIC en el aula de Educación Física: estudio a partir de parámetros de ocio digitalActive. Digital Education Review, 29, 112-123.

Chaddha, A., Jackson, E. A., Richardson, C. R., \& Franklin, B. A. (2017). Technology to help promote physical activity. American Journal of Cardiology, 119(1), 149-152. https://doi.org/10.1016/j.amjcard.2016.09.025

Chaves, I., Esquivel, J., Jiménez, A. C., \& Sánchez, H. (2018) Makey Makey and its Possible Application in Libraries. ECiencias de la Información, 8(1), 190-205.

Chien, L., \& Yu, C. (2014). Increase in physical activities in kindergarten children with cerebral palsy by employing MaKeyMaKey-based task systems. Research in developmental disabilities, 35(9), 1963-1969.

Colina, A.M. \& Túa, J.J. (2018). Apropiación de las TIC en la docencia universitaria: Statu quo ante un panorama complejo. Revista Espacios, 39(43), 1-6. Recuperado de: http:// www.revistaespacios.com/a18v39n43/18394321.html

De castro, C. (2015). Recursos educativos TIC en la enseñanza musical pianística. Revista Electrónica Complutense de Investigación en Educación Musical, 12, 37-52.

Dougherty, D. (2012). The Maker Movement. Innovations, 7(3), 11-14. Recuperado de http://www.mitpressjournals.org/doi/ pdf/10.1162/ INOV a 00135

Enriquez, A. C. (2018). La multimedia como recurso para el diseño de juguetes. Ecuador: Editorial Universidad del Azuay.

Eyles, A. M. (2018). Teachers' perspectives about implementing ICT in music education. Australian Journal of Teacher Education, 43(5), 110-131. http://dx.doi.org/10.14221/ ajte. $2018 \mathrm{v} 43 \mathrm{n} 5.8$

Fernández, R., Herrera, J. I., \& Navarro, R. (2015). Las TIC como recurso en la didáctica de la Educación Física escolar. Propuesta práctica para la Educación Primaria. EmásF: Revista digital de educación física, 35, 58-69.

Folch, C., Córdoba, T., \& Ribalta, D. (2020). La performance: Una propuesta interdisciplinar de las áreas de educación física, educación musical y educación visual y plástica en la formación inicial de los futuros maestros. Retos, 37, 613-619.

García, F., Alfaro, A., Hernández, A., \& Molina, M. (2006). Diseño de Cuestionarios para la recogida de información: metodología y limitaciones. Revista Clínica de Medicina de Familia,1(5), 232-236.

García, J. M. (2015). Robótica Educativa: La programación como parte de un proceso educativo. RED: Revista de Educación a distancia, 46(8), 1-11.

García-Varcárcel, A., \& Caballero, Y. A. (2019). Robótica para desarrollar el pensamiento computacional en Educación Infantil. Comunicar: Revista científica iberoamericana de comunicación y educación, 59, 63-72. https://doi.org/10.3916/ C59-2019-06
Giráldez, A. (2010). Repensar la educación musical en un mundo digital. En Giráldez, A. (Ed.). Música. Complementos de formación disciplinar (pp. 73-100). Barcelona: Grao.

González, J., Estebanell, M., \& Peracaula, M. (2018). ¿Robots o programación? El concepto de Pensamiento Computacional y los futuros maestros. Education in the Knowledge Society, 19(2), 29-45. https://doi.org/10.14201/ eks20181922945

Hagerman, M. (2017). Les Bricoscientifiques: Exploring the Intersections of Disciplinary, Digital, and Maker Literacies Instruction in a Franco-Ontarian School. Journal of Adolescent \& Adult Literacy, 61(3), 319-325.

Hernández, J. R., Cardona, M. C., \& Hernández, J. A. (2016). The effects of an individualised ICT-based music education programme on primary school students' musical competence and grades. Music Education Research, 18(2), 176-194. https:/ /doi.org/10.1080/14613808.2015.1049255

Hernández, R., Fernández, C., \& Baptista, M. P. (2014). Metodología de la investigación. Madrid: McGraw Hill.

Hinojo, F. J., López, J., Fuentes, A., Trujillo, J. M., \& Pozo, S. (2020). Academic Effects of the Use of Flipped Learning in Physical Education. International Journal of Environmental Research and Public Health, 17(1), 1-14. https://doi.org/ 10.3390/ijerph17010276

Hinojo, F. J., Mingorance, Á., Trujillo, J. M., Aznar, I., \& Cáceres, M. P. (2018). Incidence of the Flipped Classroom in the Physical Education Students' Academic Performance in University Contexts. Sustainability, 10(5), 1-13. https://doi.org/10.3390/ su10051334

Jason, C. W., \& Jammie, K. M. (2019). From Teacher-Designer to Student-Researcher: a Study of Attitude Change Regarding Creativity in STEAM Education by Using Makey Makey as a Platform for Human-Centred Design Instrument. Journal for STEM Education Research, 2(1), 75-91. https://doi.org/ 10.1007/s41979-018-0010-6

Jódar, J. (2010). La era digital: Nuevos medios, nuevos usuarios y nuevos profesionales. Razón y Palabra, 71(15), 1-11.

Ladrón, L., Cabero, J., \& Almagro, B. (2019). El conocimiento tecnológico, pedagógico y disciplinar del profesorado universitario de Educación Física. Retos, 36(36), 362-369.

Landero, R., \& González, M. (2006). Estadística con SPSS y metodología de la investigación. México: Trillas.

Lee, E., Kafai, Y., Vasudevan, V., \& Davis, R. (2014). Playing in the arcade: Designing tangible interfaces with MaKey MaKey for Scratch games. Playful User Interfaces, 12(3), 277-292.

Ley Orgánica 8/2013, de 9 de diciembre, para la mejora de la calidad educativa. Madrid, 10 de diciembre de 2013, num. 295, pp. 97858-97921.

Lleixà, T. (2017). Didáctica de la Educación Física: Nuevos temas, nuevos contextos. Didacticae: Revista de Investigación en Didácticas Específicas, 2, 2-5.

López, J., \& Fuentes, A. (2018). El liderazgo aplicado a los modelos diferenciados en educación: El caso de las cooperativas de enseñanza. En J. Gairín, \& C. Mercader (Eds.). Liderazgo y gestión del talento en las organizaciones. (p. 169-175). Madrid: Wolters Kluwer.

López, J., Pozo, S., Fuentes, A., \& Romero, J. M. (2019a). Nuevos aprendizajes para la sociedad del futuro: estado de inclusión de la robótica en las cooperativas de enseñanza de Andalucía, España. Revista Espacios, 40(15), 1-14.

López, J., Pozo, S., Fuentes, A., \& Romero, J. M. (2019b). Análisis del Liderazgo Electrónico y la Competencia Digital del Profesorado de Cooperativas Educativas de Andalucía (España). Multidisciplinary Journal of Educational Research, 9(2), 194-223. http://dx.doi.org/10.447/ remie.2019.4149 
López, J., Pozo, S., Vicente, M. R., \& Díaz, M. T. (2019). Herramientas robóticas para la dinamización de nuevos espacios educativos. Campus Virtuales, 8(1), 63-73.

Lozano, P. A., Guerrero, B. A., \& Gordillo, W. D. (2016). Scratch y Makey Makey: herramientas para fomentar habilidades del pensamiento de orden superior. Redes de Ingeniería, 1(7), 1623.

Mäkelä, S., \& Vellonen, V. (2018). Designing for appropriation: A DIY kit as an educator's tool in special education schools. International Journal of Human-Computer Studies, 118, 14-23. https://doi.org/10.1016/j.ijhcs.2018.05.004

Maldonado, G.A., García, J., \& Sampedro-Requena, B., (2019). The effect of ICT and social networks on university students. RIED. Revista Iberoamericana de Educación a Distancia, 22(2), 153-176. http://dx.doi.org/10.5944/ried.22.2.23178

Mcmillan, J., Schumacher, S. (2005). Investigación educativa. Madrid: Pearson

Méndez, J., \& Delgado, M. (2016). Las TIC en centros de Educación Primaria y Secundaria de Andalucía. Un estudio de casos a partir de buenas prácticas. Digital Education Review, 29, 134 165.

Monguillot, M., González, C., \& Guitert, M. (2015). Diseño de situaciones de aprendizaje mediadas por TIC en Educación Física. Revista Ibero-americana de educação, 2(68), 63-82.

Mouhaffel, A. G. (2018). Comparación de la enseñanza dos sistemas de programación robótica enfocada a los recursos matemáticos: Arduino+ Scratch y Sistema Lego EV3. International Journal of Innovation and Applied Studies, 25(1), 15-39.

Ortega, I. (2009). La alfabetización tecnológica. Teoría de la Educación. Educación y Cultura en la Sociedad de la Información, 2(10), 11-24.

Quezada, C.J., Teijeiro, M., Riofrío, O.G. \& Brito, L.F. (2018). Las TIC y su mediación en el fortalecimiento de la educación superior. Revista Espacios, 35(39), 1-6. Recuperado de: http:// www.revistaespacios.com/a18v39n43/18394321.html

Real Decreto 1105/2014, de 26 de diciembre, por el que se establece el currículo básico de la Educación Secundaria Obligatoria y del Bachillerato. Madrid, 3 de enero de 2015, num. 3, pp. 169 546.

Reche, P., Cardona, D., Díaz, A., Gómez, C. D., \& Pino, J. (2018). Demandas tácticas de juegos reducidos en fútbol: influencia de la tecnología utilizada. Revista Internacional de Medicina y Ciencias de la Actividad Física y el Deporte, 19(76), 729-744. https:/ /doi.org/10.15366/rimcafd2019.76.011

Rivilla, J., Sillero, M., Grande, I., Sampedro, J., \& Gómez M. Á. (2014). Do ICT resources improve sports teaching processes in handball?. Revista Internacional de Medicina y Ciencias de la Actividad Física y el Deporte, 14(53), 53-67.

Rodríguez, A. M., Cáceres, M. P., \& Alonso, S. (2018). La competencia digital del futuro docente: análisis bibliométrico de la productividad científica indexada en Scopus. International Journal of Educational Research and Innovation. IJERI, 10, 317-333.

Rodríguez, A.M., Romero, J.M., \& Campos, M.N. (2018). De nativos digitales a aprendices digitales: la realidad que se esconde en las universidades españolas. En E. López-Meneses, F.M. Sirignano., E. Vázquez-Cano, \& A.H. Martin-Padilla (Eds.), Innovaciones e investigaciones universitarias hispano-italianas (p. 116-132). Sevilla, España: GEFORAN.

Román, S., Almansa, A., \& Cruz, M.R. (2016). Adultos y mayores frente a las TIC. La competencia mediática de los inmigrantes digitales. Comunicar, 24(49), 101-109. http://dx.doi.org/ 10.3916/C49-2016-10

Román, P., Hervás, C., \& Guisado, J. L. (2017). Experiencia de innovación educativa con robótica en la Facultad de Ciencias de la Educación de la Universidad de Sevilla (España). En J. Ruiz,
J. Sánchez, \& E. Sánchez (Eds). Innovación docente y uso de las TIC en educación. (p. 1-16). Málaga: UMA Editorial.

Ruismäki, H., \& Juvonen, A. (2009). The new Horizons for Music Technology in Music Edu-cation. The Changing Face of Music Education. Music and Environment. Tallinn: Institute of Fine Arts.

Sáez-López, J., \& Sevillano-García, M. (2017). Sensors, programming and devices in art education sessions. One case in the context of primary education. Culture and Education, 29(2), 350-384 http://dx.doi.org/10.1080/11356405.2017.1305075

Sáez-López, J., Sevillano-García, M. \& Pascual-Sevillano, M. (2019). Aplicación del juego ubicuo con realidad aumentada en Educación Primaria. Comunicar, 61(27), 71-82. https://doi.org/ 10.3916/C61-2019-06

Sánchez, A., \& Castro, D. (2013). Cerrando la brecha entre nativos e inmigrantes digitales a través de las competencias informáticas e informacionales. Apertura, 2(5), 6-15.

Santos, D. A., Chaput, J. P., Katzmarzyk, P. T., Fogelholm, M., Hu, G., Maher, C., ... \& Tudor, C. (2018). Physical education classes, physical activity, and sedentary behavior in children. Medicine \& Science in Sports \& Exercise, 50(5), 9951004. https://doi.org/10.1249/MSS.0000000000001524

Schleicher, A. (2015). Schools for 21st-Century Learners: Strong Leaders, Confident Teachers, Innovative Approaches. International Summit on the teaching profession. OECD publishing. https://doi.org/10.1787/9789264231191

Serrano, R. M. (2017). Tecnología y educación musical obligatoria en España: referentes para la implementación de buenas prácticas. Revista Electrónica Complutense de Investigación en Educación Musical, 14, 153-169.

Suárez, Á., Specht, M., Prinsen, F., Kalz, M., \& Ternier, S. (2018). A review of the types of mobile activities in mobile inquirybased learning. Comput. Educ., 118, 38-55.

Thayer, T. (2016). Música y tecnología: taller para la integración de las TIC en el aula de educación musical. Contextos: Estudios de Humanidades y Ciencias Sociales, 27, 109-124.

Vega, D., Cufí, X., Rueda, M. J., \& Llinás, D. (2016). Integración de robótica educativa de bajo coste en el ámbito de la educación secundaria para fomentar el aprendizaje por proyectos. IJERI, $6,162-175$.

Vicente, A., \& Díaz, M.T. (2012). New challenges for Music Studies in Higher Education. Procedia Social and Behavioral Sciences, 69, 571-578

Vicente-Bújez, M.R., Díaz-Mohedo, M.T., López-Belmonte, J., \& Pozo-Sánchez, S. (2019). Educación y desarrollo de la expresión corporal y la discriminación auditiva a través de herramientas robóticas. Journal of Sport and Health Research. 11(supl 1):115-126.

Viñals, A., \& Cuenca, J. (2016). El rol del docente en la era digital. Revista Interuniversitaria de Formación del Profesorado, 30(2), 103-114.

Vivas, L., \& Sáez, J. M. (2019). Integración de la robótica educativa en Educación Primaria. Relatec, 18(1), 108-128. http:// dx.medra.org/10.17398/1695-288X.18.1.107

Weng, C., Rathinasabapathi, A., Weng, A., \& Zagita, C. (2019). Mixed Reality in Science Education as a Learning Support: A Revitalized Science Book. J. Educ. Comput. Res. 57, 777-807.

Yu-mei, D., Qing-qing, P., Jian-tong, W., Huan, D., \& Jia, M. (2017). The Practical Research on Robot Curriculum of Maker Education in Primary School. Science Journal of Business and Management, 5(3), 105-109. 